\title{
Ramírez Ludeña, Lorena. Diferencias y deferencia, Marcial Pons, Madrid, 2015.
}

Fe de rico José Arena

CONICET - U.N.C.
1. La relación entre la filosofía del lenguaje y la filosofía del derecho tiene una larga historia. La empresa de regular el comportamiento humano mediante normas es claramente una empresa comunicacional. Las normas jurídicas se encuentran paradigmáticamente expresadas en enunciados y, además, en la actualidad gran parte de ellas están fijadas en textos. Los filósofos del derecho han recurrido a la filosofía del lenguaje para abordar algunos de los problemas que la estrecha relación entre derecho y lenguaje impone $^{1}$ y también los filósofos del lenguaje han visto en el derecho un ámbito donde poner a prueba sus teorías o encontrar ejemplos ${ }^{2}$.

Sin embargo, creo que podría afirmarse que fue el libro de Herbert Hart, El concepto de derecho ${ }^{3}$, el que ubicó esa relación alrededor del concepto

\footnotetext{
${ }^{1}$ Véase, sólo para mencionar un par de ejemplos: CARRIó, 1990 [1965]; TARELLO, 1974.

${ }^{2}$ Es conocido el agradecimiento de J.L. Austin a Hart por sugerirle algunos de los ejemplos usados en Austin, 1981 [1962].

${ }^{3}$ HART, 1963 [1961].
}

mismo de derecho ${ }^{4}$. Fue luego Dworkin quien hizo explícita la relación entre teorías semánticas y concepto de derecho, que se encontraba en el trabajo de Hart. Como se sabe, Dworkin atribuye a Hart una teoría semántica criterial, según la cual el significado de un término consiste en un conjunto de criterios generalmente compartidos por una comunidad. Sobre esta base Dworkin construye una de sus más célebres objeciones al positivismo $^{5}$. Según esta objeción, si para que exista significado es necesario compartir criterios, entonces es imposible que los sujetos desacuerden acerca del significado y que, a la vez, el significado exista, i.e., estén hablando de lo

\footnotetext{
${ }^{4}$ Ello no quiere decir, claro está, que autores anteriores no se hay an referido a la naturaleza de la tarea que emprendían cuando definían el derecho. Véase, por ejemplo: "Una teoría del derecho tiene, ante todo, que determinar conceptualmente su objeto. Para alcanzar una definición del derecho, se recomienda, por de pronto, partir del uso lingüístico; es decir, establecer el significado que la palabra Recht tiene en alemán, así como sus equivalentes en otros lenguajes (law, droit, diritto, etcétera). Corresponde establecer si los fenómenos sociales designado con esa palabra exhiben notas comunes, mediante las cuales puedan ser distinguidos de otros fenómenos análogos a ellos, y si esas notas son suficientemente significativas como para servir de elementos de un concepto de conocimiento científico de la sociedad”. KELSEN, 1982 [1960]: 44.

5 Por razones de brevedad, usaré simplemente "positivismo" para referirme al positivismo jurídico.
} 
mismo. Dado que los desacuerdos son un rasgo recurrente de la práctica jurídica, esta dificultad de la semántica criterial para dar cuenta de ellos pone al positivismo frente al siguiente dilema: o bien se mantiene la semántica criterial y el positivismo, pero se dejaba sin resolver la cuestión de los desacuerdos; o bien se resuelve la cuestión de los desacuerdos apelando a otra teoría semántica, pero se abandona el positivismo.

Claramente existen diferentes modos de desarticular el dilema, es decir, de evitar resbalar por alguno de sus cuernos. Sin pretensión de exhaustividad se pueden mencionar los siguientes: primero, mostrar que la semántica criterial puede resolver el problema de los desacuerdos; segundo, mostrar que el positivismo jurídico es compatible con otras teorías semánticas; y tercero, reducir el impacto del problema de los desacuerdos 6 .

La mayoría de los defensores de Hart optaron por la primera o la tercera solución, asumiendo, quizás, que otras teorías semánticas exigirían abandonar al positivis mo ${ }^{7}$.

\footnotetext{
6 La salida que propone Dworkin es abandonar la empresa semántica y embarcarse en una interpretativa. DwORKIN, 1986: cap. II.

7 De hecho, quienes propusieron otras teorías semánticas, supuestamente capaces de hacer frente a la cuestión de los desacuerdos, lo hicieron, precisamente, para mostrar que el positivismo jurídico se encuentra equivocado. Por ejemplo, Michael Moore o Nicos Stavropoulos cuyas posiciones son ampliamente abordadas en el libro de Ramírez Ludeña.
}

Uno de los puntos originales del libro de Ramírez Ludeña es mostrar que la segunda salida es también una opción viable. Es decir, que una semántica como la defendida por las que denomina "nuevas teorías de la referencia" (de ahora en más NTR) es compatible con el positivismo y que una combinación de ambas posiciones permite dar cuenta del problema de los desacuerdos $^{8}$. Además, la autora propone recuperar la sabiduría anterior y volver a una relación más diversificada entre teoría del derecho y semántica filosófica, no concentrada exclusivamente en la discusión sobre el concepto de derecho. En este sentido, se apoya en las enseñanzas de las NTR para abordar problemas de interpretación del derecho. En particular, la autora defiende la capacidad de las NTR para "reconstruir adecuadamente el modo en que empleamos algunos términos en el ámbito jurídico" $"$.

Para llevar a cabo este programa Ramírez Ludeña estructura su obra en 5 capítulos, que pueden ser vistos como organizados en tres grandes partes. En primer lugar, repasa y sistematiza en detalle la discusión en filosofía del lenguaje, ofreciendo una formulación revisada de las NTR (capítulos 1 y 2). En segundo lugar, se ocupa de analizar cómo las posiciones no positivistas se han apoyado en las NTR y de qué modo, en cambio, una posición

\footnotetext{
${ }^{8}$ Como veremos, la estrategia de Ramírez Ludeña incluye también una reducción del impacto de los desacuerdos.

${ }^{9}$ RAMíreZ LudEÑA, 2015: 14.
} 
positivista podría aprovecharlas (capítulos 3 y 4). Finalmente, aborda el problema de los desacuerdos y propone una salida compatible con el positivismo apoyada en las NTR (capítulo 5). La estrategia de la autora es mostrar que la extendida asociación entre las NTR y posturas no positivistas acerca del derecho se debe a una incorrecta comprensión de los compromisos y tesis de las primeras. Sólo una vez que las NTR han sido adecuadamente formuladas $y$ distinguidas de otras tesis (sobre todo metafísicas) con las cuales no están comprometidas, es posible medir su incidencia en ámbito jurídico. En este sentido, la autora sostiene, primero, que las NTR son neutrales respecto de posiciones metafísicas tales como esencialismo y constructivismo. Segundo, que ello las vuelve también neutrales respecto de la discusión iusnaturalismo versus iuspositivismo. Tercero, que así concebidas son capaces además de explicar porciones de la interpretación jurídica y el fenómeno de los desacuerdos.

El libro es rico en detalles y argumentos, pero no es posible discutir todos ellos en estas breves notas. Aquí me limitaré a presentar sucintamente la versión que ofrece de las NTR y concentraré mis observaciones críticas en las tesis relativas a la incidencia de esa versión en ámbito jurídico.
2. Los capítulos 1 y 2 , titulados respectivamente 'Las nuevas teorías de la referencia frente a la concepción tradicional" y "Los elementos centrales de las nuevas teorías de la referencia", constituyen una de las mejores fuentes para un teórico del derecho interesado en abordar los problemas de filosofía del lenguaje. Quien desee embarcarse en la discusión sobre los vínculos entre semántica y teoría del derecho, estos dos capítulos constituyen una lectura indispensable. En ellos, la autora se aboca a dos tareas principales. Por un lado, poner en duda que tenga sentido una teoría semántica global, i.e., una teoría que explique el significado de todos los términos del lenguaje. Por otro lado, cuestionar el modo en que generalmente han sido entendidas las NTR. Este segundo punto me parece el más interesante y, a su vez, el más arriesgado de esta primera parte.

Una teoría de la referencia es una teoría acerca de cómo a través del lenguaje es posible capturar el mundo que nos rodea. Dicho con términos más técnicos, son teorías que persiguen explicar el carácter intensional ${ }^{10}$ del lenguaje, es decir, el hecho que mediante nuestras palabras logremos hablar acerca del mundo. Ramírez Ludeña

${ }^{10}$ Se suele convenir en escribirlo con " $\mathrm{s}$ " para advertir ya desde la grafía que no se está haciendo referencia a las intenciones. 
clasifica a estas teorías en dos grandes familias, la concepción tradicional y las $\mathrm{NTR}^{11}$.

La concepción tradicional es aquella según la cual la relación entre un término y el objeto designado se produce gracias a la intermediación de un cúmulo de descripciones de acceso público ${ }^{12}$. En cambio, para las NTR la referencia se produce directamente, sin intermediarios ${ }^{13}$.

Según las NTR, un término como "Aristóteles" no refiere a un sujeto gracias a un cúmulo de descripciones como, por ejemplo, "el maestro de Alejandro Magno", "el autor de Ética a Nicómaco", etc. Ello en cuanto, si en algún mundo posible ése mismo sujeto no fuese el maestro de Alejandro Magno ni el autor de Ética a Nicómaco, seguiríamos llamándolo Aristóteles $^{14}$. Esto sucede no sólo con los nombres propios, sino también con los términos

\footnotetext{
${ }^{11}$ La controversia entre estas familias de posiciones se estructuró, tal como se detalla en el capítulo 1, alrededor de cuatro dificultades paradigmáticas que una teoría de la referencia debería resolver: (1) El carácter informativo de algunos enunciados de identidad, (2) Los contextos opacos, (3) Los enunciados de referencia vacía y (4) Los enunciados de existencia.

${ }^{12}$ Siguiendo la literatura especializada, Ramírez Ludeña distingue dos versiones de la concepción tradicional, a saber, la clásica y la moderna. No hay espacio en esta reseña para entrar en los detalles, basta decir que la principal diferencia entre ambas es que mientras la versión clásica sostiene que se trata de un conjunto de descripciones necesarias y suficientes, la moderna sostiene que basta con que el objeto satisfaga un número razonable o suficiente, aunque indeterminado, de las descripciones asociadas al término. Véase RAmíreZ LudeÑA, 2015: 31. Representantes de la concepción tradicional son Gottlob Frege, John Searle y Peter Strawson, entre otros.

${ }^{13}$ Los principales defensores de las NTR son, como es conocido, Saul Kripke, Keith Donnellan y Hilary Putnam. La introducción de las NTR por parte de estos autores no está exenta de controversias, varias de las cuales se encuentran abordadas en el libro.

14 Este, como señala Ramírez Ludeña, es uno de los ejemplos usados por Kripke. KRIP KE, 1980 [1972].
}

de clase natural, como por ejemplo "agua". De nuevo, la referencia que ese término hace a un objeto no se produce mediante un cúmulo de descripciones como "líquido trasparente", "calma la sed", "se encuentra en los ríos y lagos", etc. Pues aún cuando un líquido satisfaga esas propiedades no lo llamaríamos "agua" si no cuenta con las propiedades estructurales del agua, i.e. $\mathrm{H}_{2} \mathrm{O}^{15}$.

Tal como señala la autora, las NTR son por lo general asociadas a las siguientes tres tesis: escencialismo ${ }^{16}$, rigidez $^{17}$ y cadena causal $^{18}$. Según este modo extendido de ver, la capacidad de los nombres propios y los de clase natural para referir directamente a un objeto, sin intermediarios descripcionales, se debe a que tales objetos poseen una esencia. En el momento en que el término fue usado por primera vez para designar el objeto, su esencia dio lugar a una cadena causal cuyos efectos son los usos sucesivos del término. Dada esa vinculación causal con las esencias, los términos designan rígidamente, es decir, designan al mismo objeto en todo mundo posible ${ }^{19}$.

\footnotetext{
${ }^{15}$ El ejemplo del agua es el usado por Putnam en su experimento mental de la tierra gemela. Putnam, 1975.

${ }^{16} \mathrm{La}$ autora entiende por esencialismo la tesis según la cual "hay sólo una forma adecuada de clasificar los objetos del mundo" RAMÍREZ LUDEÑA, 2015: 13.

${ }^{17}$ La rigidez consiste en designar al mismo objeto en todo mundo posible. Véase RAmíreZ LUDEÑA, 2015: 35.

18 Para una crítica a la idea de cadena de causalidad véase RAMÍREZ LUDEÑA, 2015: 83-85.

$19 \mathrm{La}$ autora distingue, por un lado, entre designación rígida y referencia directa y, por otro lado, entre dos formas de referencia
} 
La asociación de estas tesis con las NTR se debe, en parte, a que sus mismos defensores las han sostenido. Pero frente a estas versiones ambiciosas de las NTR, la autora propone una versión modesta ${ }^{20}$. Es decir, una versión replegada en tesis semánticas, y no comprometida con tesis metafísicas (i.e. existencia de esencias, de mundos posibles, de relaciones causales, entre otras).

Para desarticular la relación entre esencialismo y las NTR, la autora sostiene que estas últimas son teorías acerca de cómo usamos los términos y, por lo tanto, no asumen ninguna tesis metafísica robusta. Y no lo hacen porque el mundo podría haber sido diferente en varios sentidos $y$, no obstante, nosotros seguir usando los términos del mismo modo. Es decir, el mundo bien podría ser como lo concibe el esencialismo, pero también "podría ser que el mundo sea de un determinado modo, pero que existan diferentes formas igualmente correctas de clasificarlo. O, también, es concebible que el mundo esté construido por nuestras creencias y que careza de sentido hablar

directa (a) cuando no sólo los términos, sino también los objetos, contribuyen al significado y (b) cuando el vínculo entre términos y objetos no se encuentra mediado por alguna entidad. Dado que algunas descripciones pueden tanto designar de manera rígida ("el sucesor de 8 " es una descripción que designa al número 9 en todos los mundos posibles) y, además, pueden referir de manera directa en el sentido (a), Ramírez Ludeña sostiene que sólo la referencia directa en sentido (b) es diferenciadora de las NTR respecto de la concepción tradicional. Ello no obsta, a que, de hecho, los partidarios de las NTR hay an defendido tanto la designación rígida como los dos sentidos de referencia directa. Véase RAmíREZ LUDEÑA, 2015: 63.

${ }^{20}$ Las etiquetas "ambiciosa" y "modesta" no son usadas en el libro. de los objetos en sí mismos, independientemente de nuestros esquemas conceptuales" ${ }^{\prime 21}$. Todas estas posibilidades son, según la autora, compatibles con las NTR.

En definitiva, "el hecho de que podamos descubrir que no hay una única naturaleza subyacente al uso de un término no conlleva que no empleemos los términos en el sentido en que señalan los partidarios de las nuevas teorías de la referencia. Además, nada obsta a que el modo en que usamos un término pueda variar con el tiempo y las convenciones pasen a ser relevantes, lo que tampoco iría en detrimento de las nuevas teorías" 22 .

En este sentido, Ramírez Ludeña replantea la discusión entre la concepción tradicional y las NTR como una discusión acerca de la mejor reconstrucción de nuestra práctica lingüística. Desde este punto de vista, las NTR afirman que la mejor reconstrucción del uso de ciertos términos es que los "hablantes asumen que desentrañar la naturaleza de la clase resulta determinante para su aplicación correcta"23. Una vez adoptada esta lectura de las NTR, se producen ciertos movimientos en la discusión. Primero, ahora es posible que respecto de algunos términos la práctica linguística sea tal como la describe la concepción tradicional y respecto de otros tal

\footnotetext{
${ }^{21}$ RAMÍREZ LUDEÑA, 2015: 126.

${ }^{22}$ RAMÍREZ LudEÑA, 2015: 104-105.

${ }^{23}$ RAMÍREZ LUDEÑA, 2015: 126.
} 
como la describen las NTR. Es decir, ya no se asume una teoría semántica global, sino que la incidencia de cada una de estas teorías dependerá de cómo, contingentemente, se desarrolle nuestra práctica lingüística. Segundo, es necesario especificar ahora un test para determinar, respecto de cada término, cuál de las dos posiciones es una mejor explicación de la práctica en la cual el término aparece. Según la caracterización que la autora hace de la versión modesta de las NTR, el test consistiría en preguntarnos si respecto de la práctica se verifican las siguientes condiciones: (i) el cambio de creencias no supone el cambio de significado, (ii) la teorización acerca de los rasgos centrales del objeto es determinante del significado y (iii) se reconoce la posibilidad de error $^{24}$.

En definitiva, en su versión modesta, las NTR son teorías acerca de nuestras prácticas lingüísticas, es decir, acerca de cómo utilizamos ciertos términos. Según las NTR existen términos que utilizamos de un modo incompatible con la concepción tradicional ya que los usamos para hacer referencia directa a una clase (i.e., sin la intermediación de descripciones). Respecto de esos términos consideramos que su naturaleza puede trascendernos, aceptamos la posibilidad de error generalizada y creemos que es necesaria la

\footnotetext{
${ }^{24}$ Véase RAMírEZ LudEÑA, 2015: 107, 111 y 121, entre otras, para una referencia textual a este modo de caracterizar la versión modesta de NTR.
}

teorización para identificarla. Dado que las prácticas linguísticas son contingentes, cuál sea la teoría que mejor explica el uso de un término (la concepción tradicional o las NTR), depende de cómo se haya desarrollado la práctica.

3. Llegamos así a los capítulos 3 y 4, donde la autora explora las relaciones entre las NTR y la teoría del derecho ${ }^{25}$. No es difícil ver por qué la teoría de Hart ha sido asociada con la concepción tradicional. Dado que Hart sostiene que la existencia y el contenido del derecho depende de una convergencia en las actitudes y creencias de los participantes, un modo de formular esa tesis es que el significado del término "derecho" depende de cuáles sean las descripciones compartidas entre los participantes y asociadas al término ${ }^{26}$. En cambio, dado que el iusnaturalismo defiende por lo general la tesis según la cual la existencia y el contenido del derecho es independiente de las creencias de lo participantes, puede ser más

25 Titulados respectivamente: "La defensa no-positivista de las nuevas teorías de la referencia" y "Una defensa positivista de las nuevas teorías de la referencia en el derecho". No encuentro acertada la redacción de los títulos, ya que parecen ir en contra del esfuerzo de la autora por mostrar la neutralidad de las NTR respecto de estas dos posiciones.

${ }^{26}$ Una deriva de las respuestas a la objeción de Dworkin, tal como señala la autora, es o bien negar que Hart esté hablando del significado del término "derecho", sino del concepto de derecho; o bien negar que su semántica sea criterial, sino de paradigmas. Quizás estas respuestas puedan reducir la incidencia de las teorías semánticas, pero ciertamente no resuelven la objeción de Dworkin. Siempre que el contenido del significado o del concepto dependa de que exista entre los participantes un acuerdo (o convergencia), su objeción, creo, se mantiene en pie. Es decir, exige una respuesta. Es por ello que no entro aquí en las precisiones que la autora hace respecto de la distinción "significado" vs. "concepto". 
fácilmente asociado a las $\mathrm{NTR}^{27}$. De hecho, los autores abordados en el libro, a saber, Michael Moore, David Brink y Nicos Stavropoulos han defendido una posición no-positivista apoyándose en las NTR. Aquí no podré entrar en los detalles del análisis crítico que Ramírez Ludeña hace de cada uno de ellos ${ }^{28}$.

$\mathrm{Si}$ bien estos tres autores comparten la defensa de las NTR y una posición no positivista, la autora señala una diferencia relevante: "tanto Brink como Stavropoulos adoptan como elementos fundamentales de su análisis de la naturaleza del derecho el modo en que empleamos los términos y nuestras asunciones al hacerlo, mientras que Moore sitúa el énfasis en el mundo"29.

Como vimos, Ramírez Ludeña sostiene que las NTR no están necesariamente asociadas al esencialismo $\mathrm{y}$ en esto se acerca a Brink $\mathrm{y}$ Stavropoulos $^{30}$. Pero la autora objeta además la asociación entre las NTR y el iusnaturalismo. El razonamiento que está detrás de estas disociaciones parece ser el siguiente: si las NTR

${ }^{27} \mathrm{Si}$ bien en diferentes lugares hay referencia a las tesis de ambas escuelas, se extraña en el libro una precisión temprana y explícita de las tesis atribuidas al "positivismo jurídico" y a la "teoría del derecho natural".

${ }^{28}$ En el libro se discute la posición de estos autores tanto respecto de la naturaleza del derecho como respecto de la interpretación jurídica. Abordar las complejidades del argumento exigiría escribir un artículo aparte.

${ }^{29}$ RAMÍREZ LuDEÑA, 2015: 121.

${ }^{30}$ De hecho, comparte con Brink la idea según la cual "las nuevas teorías de la referencia constituyen posiciones estrictamente semánticas" y con Stavropoulos el énfasis en las prácticas linguísticas. Con este último desacuerda respecto del carácter universal de las NTR. Véase RAmírez LudEÑA, 2015: 123. estuvieran asociadas al esencialismo, entonces, contrariamente a lo que sostiene el positivismo, la existencia y el contenido del derecho sería independiente de nuestras creencias y esta última es una tesis ciertamente iusnaturalista.

Sin embargo, desde mi punto de vista, este razonamiento va demasiado rápido. Para advertir la relación entre las NTR y el positivismo es imprescindible realizar algunas distinciones ulteriores. Cuando se discute de realismo y antirrealismo, siempre vale la pena especificar el ámbito o dominio de discusión. Así, por ejemplo, asumamos que discutimos sobre normas jurídicas. La autora defiende que las NTR son compatibles con el esencialismo y el constructivismo, es decir, con diferentes formas de realismo, y esto parece sensato. Es decir, las NTR son compatibles con aquellas posiciones que sostienen que existen las normas, si bien no estén de acuerdo sobre su naturaleza $^{31}$. No obstante, no parece que las NTR sean compatibles con el antirrealismo, i.e., con posiciones que nieguen la existencia de normas jurídicas. Frente a esta objeción, el argumento de Ramírez Ludeña es que las NTR no incluyen tesis metafísicas, sino que se limitan a explicar nuestras prácticas lingüísticas. Sin embargo, si las NTR son entendidas de este modo, entonces su reconstrucción de la práctica lingüística según la

\footnotetext{
${ }^{31} \mathrm{El}$ realismo puede ser naturalista, no-naturalista o constructivista (si bien esta última opción suele ser objeto de controversia). Véase, para formas de realismo abordadas respecto del ámbito moral, SAYRE-McCord, 2006.
} 
cual tratamos ciertos términos como referidos a la esencia de objetos que no existen, las transformaría en una teoría del error. Es decir, o bien las NTR se comprometen con el realismo (ya sea esencialista o constructivista) o bien se transforman en teorías del error.

Ahora bien, ¿esto implica que las NTR son incompatibles con el positivismo? Dicho de otro modo, ¿las NTR asociadas al positivismo serían inevitablemente teorías del error? Bueno, esto depende de qué posición asuma el positivismo respecto de las normas jurídicas. Y aquí creo que deben distinguirse dos versiones del positivismo. Por un lado la versión normativista, defendida por autores como Hart y Kelsen, según la cual las normas jurídicas no pueden ser reducidas a entidades de otro tipo aun cuando, en parte, sean hechos sociales $^{32} \mathrm{y}$, por otro lado, la versión empirista $^{33}$, defendida por autores como Ross, según la cual las normas jurídicas son reducibles a hechos sociales, e.g., las creencias y actitudes de los jueces. Desde mi punto de vista, el positivismo normativista es claramente realista respecto de las normas jurídicas $\mathrm{y}$, en principio, compatible con las NTR sin transformarlas en teorías del error. El problema es que, por vía de otros argumentos, tales como, por ejemplo, la unidad del

\footnotetext{
${ }^{32}$ Véase, para una formulación explícita de esta tesis, HART, 1983 [1954].

33 El término más adecuado sería positivismo realista, en referencia al "realismo jurídico", pero para evitar confusión terminológica usaré empirista.
}

razonamiento práctico, el realismo acerca de las normas jurídicas llevaría a esta versión del positivismo a colapsar con tesis inevitablemente iusntauralistas $^{34}$. El punto importante es que, en este caso, la vinculación entre realismo y iusnaturalismo no se sigue de defender o no las NTR, sino que se debe a que los positivistas normativistas no han logrado mostrar que los hechos sociales sean normas y que no son normas morales ${ }^{35}$.

El positivismo empirista, en cambio, no es realista acerca de las normas jurídicas, pero sí acerca de los hechos sociales a los cuales esas normas son reducibles y, por lo tanto, sería compatible con las $\mathrm{NTR}^{36}$. Ello es así si distinguimos entre dos roles de las creencias. Por un lado, las creencias pueden ser las determinantes de la referencia del término "derecho" y esto sería incompatible con las NTR. Pero, por otro lado, puede ser que el término "derecho" refiera directamente a nuestras

${ }^{34}$ Un modo de reconstruir este punto es el siguiente. Así como el mundo de los hechos empíricos es uno solo y por lo tanto una proposición es verdadera o es falsa, también el mundo de los hechos normativos es uno solo y por lo tanto una norma es verdadera o es falsa. Si esto es así, entonces es posible aceptar sólo uno mundo normativo. Dicho más precisamente, si las condiciones de verdad de las normas jurídicas son hechos normativos, entonces estos han de ser necesariamente hechos morales y, por lo tanto, las normas jurídicas serán siempre, en última instancia, normas morales. Para una presentación de este argumento y una propuesta de respuesta véase CARACCIOLO, 2000: 39.

35 Véase CARACCiolo, 2000 para una defensa del positivismo frente a esta objeción.

36 Por ejemplo, según Celano, la posición positivista es antirrealista (respecto de las normas), pero las razones de ese antirrealismo impiden defender un antirrealismo global, ya que el positivista se compromete con el realismo respecto, al menos, de los hechos naturales, i.e., las creencias humanas de las cuales depende la existencia del derecho. Celano, 1999: 237-239. Véase también Moreso Mateos, 1999: 316. 
creencias. En este caso, las creencias no desempeñarían el rol de descripciones definidas, sino de objeto referido. Es decir, un defensor de las NTR podría ser realista acerca de las creencias, i.e. podría defender que el contenido y la existencia de las creencias de un grupo es independiente del contenido y la existencia de las creencias del observador, y sin embargo sostener que el término "derecho" no hace referencia a un conjunto de normas naturales o puestas por una entidad divina, sino a un conjunto de creencias de un grupo de individuos. Sin embargo, si resulta que los hablantes usan el término como refiriendo a normas, entonces el positivista empirista quedará comprometido con una teoría del error.

Resumiendo, en su versión modesta, defendida por la autora, las NTR son compatibles con el antirrealismo al precio de convertirse inevitablemente en una teoría del error. En este caso, las NTR dirán que en nuestras prácticas lingüísticas tratamos a ciertos términos como haciendo referencia directa a una clase que nos trasciende, pero que no existe. Para evitar recaer por defecto en una teoría del error, las NTR tienen que asumir alguna forma de realismo. Bajo esta versión robusta, las NTR son, en principio, compatibles tanto con el positivismo normativista, como con el positivismo empirista. Pero si aceptamos que no es posible ser realista acerca de las normas jurídicas sin colapsar con el iusnaturalismo, entonces la única forma positivista que es compatible con las NTR, sin colapsar con el iusnaturalismo, es el positivismo empirista. Sin embargo, que este último quede o no comprometido con una teoría del error dependerá de cómo contingentemente se haya desarrollado la práctica.

4. Como adelanté, Ramírez Ludeña no sólo persigue mostrar la compatibilidad entre las NTR y el positivismo, sino que parte de su empresa es también mostrar que las teorías semánticas son mejor entendidas como empresas locales. Es decir, como empresas destinadas a explicar porciones de nuestras prácticas lingüísticas, sin pretensiones de universalidad. Por ello, separa las tesis acerca del concepto de derecho de las tesis sobre la interpretación de textos normativos y de la discusión sobre los desacuerdos. Terminaré entonces esta reseña refiriéndome a estos dos puntos, empezando por la interpretación.

La autora se preocupa sobre el final del capítulo 4 de discutir la relación entre las NTR y algunas posiciones en teoría de la interpretación. En particular se refiere a la relación entre las NTR y la relevancia que, en la interpretación, se otorga a las intenciones del legislador, a la autoridad del derecho y al principio de legalidad. Estos tres rasgos son presentados como una incomodidad para las NTR. La estrategia de la autora es, primero, diluir la relevancia de estos rasgos $\mathrm{y}$, segundo, mostrar que una vez diluidos no generan 
ninguna incomodidad. Para no extender demasiado estas ya poco breves notas, me referiré aquí solo a la discusión de la intención del legislador (considero, además, que lo que diré al respecto puede extenderse, con ciertos ajustes, a la discusión de la autoridad del derecho y el principio de legalidad).

La intención del legislador resulta, se alega, incómoda para quien desee aplicar las NTR al análisis de la interpretación jurídica porque, si el significado está determinado por el mundo, entonces el legislador puede equivocarse al referir $\mathrm{y}$, en ese caso, las NTR nos impondrían atribuir a la disposición un significado que el legislador no tenía intención de comunicar. En realidad, a primera vista, parecería que la respuesta más fácil para los defensores de las NTR es que la deferencia a las intenciones del legislador es un problema para todas las teorías del significado que no sean equivalentes a "la disposición normativa significa lo que el legislador tenía la intención que signifique". Es decir, el significado literal, el significado teleológico, el significado sistemático, el significado que sea será siempre potencialmente diferente al atribuido por el legislador. Incluso más, dado que el legislador, si pretende comunicar, no puede pretender que el significado de todas las disposiciones es el que tenía la intención que sea, el problema es inevitable.
Sin embargo, Ramírez Ludeña no elige esta respuesta, sino que cuestiona la noción de intenciones del legislador buscando diluir el impacto de la objeción. Mediante argumentos que denomina conceptuales apunta a mostrar las diferentes dificultades para identificar la intención del legislador: pluralidad de sujetos, opacidad, niveles de abstracción, entre otros. Y, sobre esa base, sostiene que, a la hora de identificar el significado de las disposiciones normativas, es preferible optar por las NTR en lugar de las intenciones del legislador. Dadas las incertidumbres señaladas respecto de la intención del legislador, si nos interesa en alguna medida preservar la previsibilidad en la interpretación, "parecería que ésta se garantiza en mayor medida si el juez busca y fundamenta la solución en atención a la naturaleza de la clase en cuestión que si trata de reconstruir la intención del legislador, con todos los problemas que ello trae aparejado"37.

Sin embargo, no parece que esta defensa normativa sea la que necesitan las NTR para considerarlas una buena explicación de una práctica interpretativa en particular. Creo que el problema más grave para las NTR, tal como las entiende la autora, es que la relevancia de las intenciones del legislador no encaja con la explicación que proponen de la práctica linguística. Como ha sido señalado, bajo la

${ }^{37}$ RAMíREZ LudEÑA, 2015: 147. 
versión de Ramírez Ludeña, las NTR son una reconstrucción de nuestras prácticas lingüísticas. Según esta reconstrucción, el significado de algunos términos está en parte determinado por el objeto al que refieren $\mathrm{y}$, por lo tanto, ante situaciones contrafácticas sostendríamos que las intenciones de los hablantes son irrelevantes. El problema es que en ámbito jurídico, en cambio, las intenciones del legislador sí son relevantes para la interpretación. Por lo tanto, no se satisface la primera exigencia del test, ya que en estos casos tratamos al significado como dependiente de las creencias. Si ello es así, parece que no tratamos los términos jurídicos como los tratarían sujetos involucrados en una práctica linguística tal como la conciben las $\mathrm{NTR}^{38}$.

En el último capítulo, titulado 'Los desacuerdos en el derecho", la autora discute la objeción basada en los desacuerdos dirigida por Dworkin contra el positivismo. Allí ensaya, como adelanté, una reducción del impacto de la objeción recurriendo a diferentes estrategias. Entre ellas, restringir la cantidad, ubicación y alcance de los desacuerdos e introducir algunas sofisticaciones a la semántica criterial $^{39}$. Una vez delimitada la porción del fenómeno jurídico que quedaría sin

\footnotetext{
${ }^{38}$ Este argumento puede ser, estimo, extendido a aquellos casos en que al interpretar se considera relevante la autoridad de quien emite la norma y el respeto al principio de legalidad. Aunque ello exigiría abordar en detalle las diferentes estrategias que en cada caso propone la autora.

39 En particular la autora aborda las ideas desarrolladas bajo la etiqueta de "convencionalismo profundo" por Juan Carlos Bayón. Véase BAYÓn MoHINo, 2002.
}

explicar, la autora propone una original distinción entre niveles de desacuerdo y adelanta su respuesta "pluralista", en el sentido que para cada nivel de desacuerdo se ofrece una respuesta específica $^{40}$. Aquí me concentraré sólo en la incidencia que, según la autora, las NTR tienen respecto de la objeción. Las NTR son relevantes respecto de un tipo particular de casos, a saber, aquellos en que "se discute acerca del significado de un término de modo que los diversos intervinientes en la disputa presentan concepciones en pugna acerca de los rasgos centrales del objeto al que el término refiere" 41 . Estos casos son problemáticos porque según el positivista el significado de las disposiciones depende de que haya convergencia, pero la existencia de desacuerdo prueba la ausencia de ella y por lo tanto la ausencia de significado. Los desacuerdos acerca del significado serían entonces imposibles, i.e., los involucrados estarían hablando de cosas diferentes. Sin embargo, modificando la idea de convergencia por la de referencia directa, los desacuerdos se volverían inteligibles. A pesar de los desacuerdos, somos capaces de referir porque "formamos parte de una cadena de comunicación, que nos remonta en última instancia a ejemplares de la clase,

\footnotetext{
40 Los niveles de desacuerdo son: (i) relativos al derecho en general: metodológico, sobre los elementos centrales del derecho, sobre la interpretación en abstracto, (ii) relativos a sistemas jurídicos específicos: sobre la identificación del derecho, sobre las fuentes jurídicas (como token y como type), sobre el significado de las fuentes, sobre la respuesta para un supuesto específico.

${ }^{41}$ RAMíreZ LudEÑA, 2015: 177.
} 
aunque nuestras creencias respecto a los objetos que pertenecen a la clase sean muy pobres y falibles. El uso correcto del término dependerá de cuál es la naturaleza de la clase a que referimos, y la identificación de qué rasgos son determinantes puede estar en manos del hablante común o de

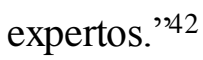

Los desacuerdos pueden ser vistos entonces como una discusión acerca de cuál es la cadena comunicativa relevante (la del lenguaje ordinario o la del lenguaje técnico), acerca de cuál es la opinión de los expertos, y, cuando estos últimos también desacuerdan, acerca de cuál es la naturaleza del objeto. Según Ramírez Ludeña, todas estas respuestas son posibles bajo el paraguas de las NTR y compatibles con el positivísimo, ya que no suponen visiones enfrentadas sobre los fundamentos del derecho, "sino que se limitan a disputas relativas a la naturaleza de un determinado objeto al que refieren las disposiciones jurídicas" ${ }^{" 43}$.

Estas tesis acerca del desacuerdo (pero también las referidas a la interpretación) persiguen mostrar la viabilidad de un teoría semántica local (o no global). En el sentido de que es posible proponer una teoría semántica para algunos términos que figuran en las disposiciones jurídicas sin que ello implique proponerla para todos ni, mucho menos,

\footnotetext{
${ }^{42}$ RAMíreZ LudEÑA, 2015: 178.
}

${ }^{43}$ RAMíREZ LudeÑA, 2015: 181. proponerla para el término "derecho". A la base de esta posición se encuentran los argumentos de Ramírez Ludeña a favor de una versión modesta de las NTR (i.e., sin compromisos metafísicos).

5. En conclusión, a lo largo de sus 5 capítulos el libro Diferencias y deferencia ofrece una detallada presentación de las relaciones entre filosofía del lenguaje y teoría del derecho. Desmenuza críticamente no sólo las posiciones de los juristas, sino también la de los filósofos del lenguaje. Una de las virtudes del texto es precisamente ofrecernos las herramientas teóricas para entender los encendidos debates acerca del concepto del derecho, la interpretación jurídica y el problema de los desacuerdos. En ese sentido, el libro es una excelente defensa de la relevancia de las "gramáticas" frente a escépticos como la Reina Isabel.

\section{Bibliografia}

J.L. Austin, 1981 [1962]: Cómo hacer cosas con palabras, G.R. CARRIÓ Y E.A. RABOSSI (Trads.). Buenos Aires: Paidós.

J.C. BAYÓN MOHINO, 2002: «El contenido mínimo del positivismo jurídico». en V. ZAPATERO GómEZ (ed.), Horizontes de la filosofía del derecho: homenaje a Luis García San Miguel, Vol. 2. Alcalá de Henares: Universidad de Alcalá de Henares.

R. CARACCIOLO, 2000: «Realismo Moral vs. Positivismo Jurídico», Analisi e diritto:37-44. 
G.R. CARRIÓ, 1990 [1965]: Notas sobre derecho y lenguaje. Buenos Aires: Abeledo - Perrot.

B. Celano, 1999: «Efficacia, anti-realismo, interpretazione», Analisi e diritto, 1999: 229247.

R. Dworkin, 1986: Law's Empire. Cambridge London: Belknap Press of Harvard University Press.

H.L.A. HART, 1963 [1961]: El concepto de derecho, G. CARrió (Trad.). Buenos Aires: AbeledoPerrot.

H.L.A. HART, 1983 [1954]: «Definition and Theory in Jurisprudence». en H.L.A. HART (ed.), Essays in Jurisprudence and Philosophy. Oxford: Clarendon Press.

H. KELSEN, 1982 [1960]: Teoría pura del derecho, R.J. VERnENGo (Trad.). México D.F.: U.N.A.M.
S. KRIPKE, 1980 [1972]: Naming and Necessity. Oxford, UK: Basil Blackwell.

J.J. MoReso MAteos, 1999: «De nuevo sobre la vigilia. A modo de réplica a mis críticos», Analisi e diritto, 1999: 295-341.

H. Putnam, 1975: «The Meaning of Meaning». en H. Putnam (ed.), Mind, Language and Reality. Cambridge: Cambridge University Press.

L. RAMÍREZ LUDEÑA, 2015: Diferencias y deferencia. Sobre el impacto de las nuevas teo rías de la referencia en el derecho. Madrid: Marcial Pons.

G. SAYRE-MCCORD, 2006: «Moral Realism». en D. CoPP (ed.), The Oxford Handbook of Ethical Theory. New York: Oxford University Press.

G. TAREllo, 1974: Diritto, enunciati, usi: studi di teoria e metateoria del diritto. Bologna: Il mulino. 